\title{
Progress in adjuvant treatment of melanoma patients
}

\author{
Piotr Rutkowski ${ }^{1}$, Wojciech M. Wysocki ${ }^{2}$, Tomasz Świtaj ${ }^{1}$, Arkadiusz Jeziorski ${ }^{3}$
}

Surgery is therapy of choice in melanoma patients. However, prognosis of patients at stage IIC-IV even after radical resection is very heterogenous and related to high risk of disease relapse. Positive results of clinical trials indicate that in the nearest future systemic adjuvant therapy in high risk melanomas will become the standard of care. New treatment modalities, both molecular targeted therapy with BRAF+MEK inhibitors dabrafenib with trametinib and immunotherapy anti-PD-1 with nivolumab or pembrolizumab have been approved in US and EU.

NOWOTWORY J Oncol 2018; 68, 3: 140-145

Key words: melanoma, adjuvant therapy, BRAF, MEK inhibitors, immunotherapy, anti-PD-1

Surgery is therapy of choice in melanoma patients, however, prognosis in patients at stage IIC-IV melanomas even after radical resection is very heterogenous and to a large extent related to high risk of disease relapse [1-5].

Adjuvant therapy after surgical treatment is currently used in specific cases, although studies indicate that systemic adjuvant therapy in the discussed group of melanoma patients will become the standard of care. New systemic therapies have already been registered in the United States and the European Union. In view of the combination of surgery and conservative treatment, the basic and binding principle should be the management by multi-specialist teams, whose members have experience in the diagnosis and treatment of patients with melanoma at locoregional and generalised settings.

\section{Adjuvant radiotherapy}

In individual cases, after surgery in patients with high risk melanoma, adjuvant radiotherapy (RTH) is possible the dosing regiment includes hypofractionation using 3-8 Gy/fraction or conventional fractionation depending on the location. The indications for adjuvant RTH after primary tumour excision may include:
- diagnosis of desmoplastic melanoma excised with narrow margins,

- the presence of 'positive' surgical margins (especially after the local recurrence resection) while no surgical radicalisation is possible,

- the presence of satellite lesions,

- enhanced neurotropism,

- location in the head and neck region (caution: RTH as an exclusive treatment method can be used with extensive LMM lesions).

In case of local resection and lymphadenectomy due to metastases at regional lymph nodes, the indications for complementary RTH may be:

- the presence of extracapsular node invasion,

- spread to $\geq 4$ lymph nodes (stage IIIC)

- metastasis diameter $>3 \mathrm{~cm}$,

- metastases in the neck lymph nodes (from 2 metastatic lymph nodes or at a minimum metastasis size of $2 \mathrm{~cm}$ ),

- relapse after prior resection [1, 2, 4, 6].

The results of the only completed randomized clinical trial , which evaluated the value of adjuvant radiotherapy (48 Gy in 20 fractions) after lymphadenectomy in case of high risk of relapse, confirmed the improvement of local

${ }^{1}$ Department of Soft Tissue/Bone Sarcoma and Melanoma, Maria Skłodowska-Curie Institute — Oncology Center, Warszawa, Poland

${ }^{2}$ Department of Surgical Oncology, Maria Skłodowska-Curie Institute - Oncology Center, Kraków Branch, Poland ${ }^{3}$ Second Department of Surgical Oncology, Nicolaus Copernicus Voivodship Multidisciplinary Center for Oncology and Traumatology, Łódź, Poland 
control after irradiation, but at the same time no effect on overall survival was observed, with an increase in frequency of late locoregional complications and deterioration of patients' quality of life. Therefore, the conclusions from this study indicate that the use of adjuvant radiotherapy should be limited $[7,8]$. It should also be emphasized that there are no indications of an adjuvant RHT after the completion lymphadenectomy following a positive sentinel lymph node biopsy (CLND)

\section{Systemic adjuvant therapy}

At present, there are no Polish and European recommendations for routine systemic adjuvant therapy in clinical practice at patients after the radical resection of primary lesions and lymphadenectomy, and adjuvant radiotherapy can only be considered in the specific situations described above. The results of recently published clinical studies indicate an improvement in survival, both from the use in adjuvant therapy the immunotherapy with the immune checkpoint inhibitors and from the combination therapy with BRAF and MEK inhibitors, and it can be expected that such treatment will become a standard of care in the nearest future, as it happened in US [1-4].

\section{Interferon}

For many years, apart from interferon (IFN), no other agents has been effective in the treatment of high risk skin melanomas. Interferon (mainly IFN-a-2b, used only in monotherapy) in the adjuvant therapy of melanoma patients (for a selected group) in most studies resulted in prolongation (in a repetitive manner) of relapse-free survival (RFS) (Tab. I) [4, 9-13]. The evidence of improved overall survival (OS) as a result of the use of IFN is much weaker and more controversial. In 10 out of 17 evaluated studies an improvement in RFS was observed, with the latest meta-analyses showing a reduction in the risk of relapse by $17-18 \%$ [relative risk (HR hazard ratio) 0.82-0.83; $\mathrm{p}<0.0001$ ] when using IFN in adjuvant therapy. The evidence for OS improvement comes mainly from meta-analyses and it is reflected in the improvement of OS by about 3\% over 5 years in the whole group of patients. The use of adjuvant IFN treatment in all high-risk melanoma patients is therefore not justified (especially considering its high toxicity) and remains optional only for selected patients. Interferon $\mathrm{a}-2 \mathrm{~b}$ (IFN a-2b) in high doses was registered in the United States and the European Union for IIB-III stage melanomas on the basis of a positive result of one of three studies of the Eastern Cooperative Oncology Group (ECOG) - ECOG 1684, while this drug was registered in low doses in Europe for II stage patients. The basis for registration was a significant increase in the overall survival time during the period of about 7 years of observation, which, however, after a longer period (12 years) was not confirmed. The results of meta-analyses indicate that the basic group of patients benefiting from IFN adjuvant therapy are those with ulcerated primary melanoma, especially in the subgroup of patients with clinically undetectable metastases in the sentinel node (formerly known as micro-metastases), and not with clinically diagnosed metastases found in swollen (palpable) lymph nodes (formerly known as macro-metastases) $[11,12]$. Results of a study by the European Organisation for Research and Treatment of Cancer 18081 (EORTC 18081) are currently expected; its aim is to assess the use of pegylated IFN in the treatment of patients after primary ulcerated cutaneous melanoma resection without metastases to regional lymph nodes (interrupted study recruitment). The most common side effects are parainfluenza symptoms, fever, weakness, neutropenia, hepatotoxicity and depression. Part of the IFN toxicity profile changes during therapy. Along the course of treatment, parainfluenza symptoms decrease, while other reported side effects remain the same or even increase with the duration of treatment (mainly fatigue, anorexia, symptoms of depression/anxiety).

\section{Immunotherapy with immune checkpoint inhibitors}

In 2015, preliminary results of a study on the use of adjuvant treatment with anti-CTLA-4 antibodies (ipilimumab) after lymphadenectomy due to metastases to regional lymph nodes (stage III) were published. The study included 951 patients who were randomly allocated to the ipilimumab high-dose group at $10 \mathrm{mg} / \mathrm{kg}$ body weight every 3 weeks and then every 3 months up to 3 years $(n=476)$ or placebo $(n=476)$. With a median of the observation time being 2.7 years, 234 events were reported with reference to RFS in ipilimumab-treated group compared to 294 for placebo-treated group; the median RFS was 26.1 months compared to 17.1 months ( $p=0.0013$ ), respectively. The improvement of RFS referred to patients with both macro- and micro-metastases (definitions according to then valid $7^{\text {th }}$ revision of TNM staging system) to lymph nodes; the result of adjuvant therapy was more important at the ulceration of the primary lesion. In the ipilimumab-treated group, $54 \%$ of patients had side effects with 3-4 toxicity levels compared to $25 \%$ in the placebo-treated group. Due to complications connected with ipilimumab administration, 5 patients (1\%) died. Side effects led to permanent discontinuation of treatment in $52 \%$ of patients entering ipilimumab treatment [14]. The results of this study, presented in 2016 with a median follow-up time at 5.3 years, show a significant improvement in the use of ipilimumab adjuvant therapy in high doses for both RFS as well as distant metastasis free survival and OS. The percentage of 5 -year OS in the group receiving ipilimumab was $65.4 \%$ compared to $54.4 \%$ in the group receiving placebo (hazard ratio for death $0.72,95.1 \%$ $\mathrm{Cl} 0.58-0.88 ; \mathrm{p}=0.001$ ) [15]. Preliminary results of a subsequent E1609 study showed similar efficacy of a lower dose 
Table I. Summary of the results of the most important clinical trials on adjuvant therapy with interferon (INF). Bold typeface distinguishes the studies where a significant benefit was obtained from INF therapy [acc. to 13]

\begin{tabular}{|c|c|c|c|c|}
\hline Study & $\begin{array}{l}\text { Study period/ } \\
\text { INF type }\end{array}$ & $\begin{array}{l}\text { Melanoma } \\
\text { stage }\end{array}$ & RFS & OS \\
\hline \multicolumn{5}{|c|}{ Very low doses of interferon, $0.5-1$ million IU } \\
\hline EORTC 18871/DKG-80 & $\begin{array}{l}1 \text { year/ } \\
\text { INF-a-2b }\end{array}$ & $\| \mathrm{B}, \mathrm{II}$ & $p=0.02$ & $p=0.18$ \\
\hline \multicolumn{5}{|c|}{ Low doses of interferon, 3 million IU } \\
\hline Austria & $\begin{array}{l}1 \text { year/ } \\
\text { INF-a-2a }\end{array}$ & $\| \mathrm{B}, \mathrm{II}$ & $p=0.02$ & $p=0.6$ \\
\hline France & $\begin{array}{l}18 \text { months/ } \\
\text { INF-a-2a }\end{array}$ & $\| \mathrm{B}, \mathrm{II}$ & $\begin{array}{l}\text { Benefit } \\
(p=0.035)\end{array}$ & $p=0.06$ \\
\hline WHO-16 & $\begin{array}{l}3 \text { years/ } \\
\text { INF-a-2a }\end{array}$ & III & $p=0.5$ & $p=0.7$ \\
\hline E1690 & $\begin{array}{l}2 \text { years/ } \\
\text { INF-a- } 2 b\end{array}$ & $\| \mathrm{B}, \mathrm{II}$ & $p=0.17$ & $p=0.81$ \\
\hline Scotland & $\begin{array}{l}6 \text { months/ } \\
\text { INF- } a-2 b\end{array}$ & $\| \mathrm{B}, \mathrm{II}$ & $p=0.051$ & $p=0.4$ \\
\hline Germany & $\begin{array}{l}2 \text { years/ } \\
\text { INF-a- } 2 a\end{array}$ & III & $p=0.0045$ & $p=0.018$ \\
\hline UKCCCR & $\begin{array}{l}2 \text { years/ } \\
\text { INF-a-2a }\end{array}$ & $\| \mathrm{B}, \mathrm{\| l}$ & $p=0.3$ & $p=0.6$ \\
\hline \multicolumn{5}{|c|}{ Intermediate doses of interferon, 10 million IU } \\
\hline \multirow[t]{2}{*}{ EORTC 18952} & $\begin{array}{l}13 \text { months/ } \\
\text { INF- } a-2 b\end{array}$ & $\| \mathrm{B}, \mathrm{II}$ & Benefit only in subgroup IIB & No benefit \\
\hline & $\begin{array}{l}25 \text { months/ } \\
\text { INF- } a-2 b\end{array}$ & $\|B\|$, & $\begin{array}{l}\text { Benefit only in the IIB subgroup or } \\
\text { in the group of patients with micro- } \\
\text { metastases after a positive sentinel } \\
\text { lymph node biopsy }\end{array}$ & No benefit \\
\hline \multicolumn{5}{|c|}{ High doses of interferon, 20 million $\mathrm{IU} / \mathrm{m}^{2}$ vs observation } \\
\hline E1684 & $\begin{array}{l}52 \text { weeks/ } \\
\text { INF-a-2b }\end{array}$ & $\|B\|$, & $p=0.02$ & $p=0.01$ \\
\hline E1690 & $\begin{array}{l}52 \text { weeks/ } \\
\text { INF- } a-2 b\end{array}$ & $\| \mathrm{B}, \mathrm{II}$ & No benefit & No benefit \\
\hline NCCTG 83707 & $\begin{array}{l}3 \text { months/ } \\
\text { INF-a-2a }\end{array}$ & $\|A\| B,, I \|$ & $p=0.24$ & $p=0.53$ \\
\hline Sunbelt Melanoma Trial & $\begin{array}{l}52 \text { weeks/ } \\
\text { INF- } a-2 b\end{array}$ & IIIA & No benefit & No benefit \\
\hline \multicolumn{5}{|c|}{ High doses of interferon, 20 million $\mathrm{IU} / \mathrm{m}^{2}$ vs vaccine } \\
\hline E1694 & $\begin{array}{l}96 \text { weeks/ } \\
\text { INF-a-2b vs GM }\end{array}$ & $\| \mathrm{B}, \mathrm{II}$ & $p=0.0015$ & $p=0.009$ \\
\hline \multicolumn{5}{|c|}{ Long-term administration of pegylated interferon vs observation } \\
\hline EORTC 18991 & & & $\mathbf{p}=\mathbf{0 . 0 1}$; improvement by $6.7 \%$ & $p=0.78$ \\
\hline
\end{tabular}

RFS - relapse-free survival; OS — overall survival

of ipilimumab ( $3 \mathrm{mg} / \mathrm{kg}$ ) with less toxicity. The EORTC 18071 study has led to the registration of ipilimumab in the United States as an adjuvant therapy for melanoma patients after lymphadenectomy due to metastases to regional lymph nodes, however, its practical application is limited due to its high toxicity and more favourable test results with anti-PD-1 antibodies (nivolumab and pembrolizumab) and kinase inhibitors.
Nivolumab in a randomized clinical trial (CheckMate 238) in patients after resection of metastases at stage IIIB, IIIC and IV showed a $10 \%$ improvement in relapse-free survival if compared with ipilimumab at a lower toxicity (18-month RFS: $65 \%$ vs $53 \%$ ). This is the only study where patients after the resection of distant metastases were also included. The improvement of distant metastases free survival (DMFS) was also demonstrated (HR 0.73). Adverse events associated with treatment at stage III or IV were reported 


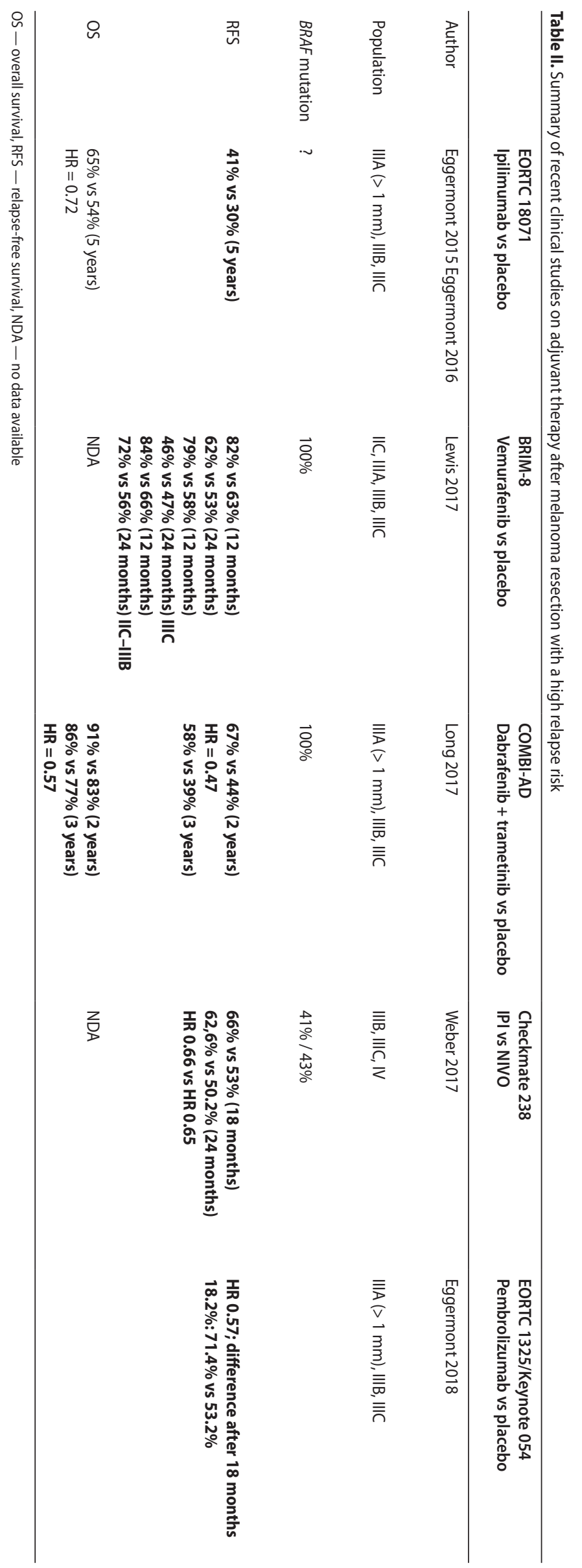


in $14.4 \%$ of patients receiving nivolumab as compared to $45.9 \%$ in the ipilimumab-treated group [16]. Updated data from 2018, with a longer period of observation, confirm the beneficial effect of nivolumab in adjuvant therapy for one year, regardless of the PD-L1 expression status and BRAF mutation with respect to RFS (HR 0.66) and DMFS (HR 0.76) [17]. Nivolumab is currently registered for adjuvant therapy in the United States and the European Union.

Preliminary results of Keynote-054/EORTC 1325 trial in 1019 patients also indicate a reduction in the risk of relapse (HR for RFS 0.57) and DMFS using pembrolizumab adjuvant therapy for one year compared to placebo in the group of patients with resection stage III with a higher risk (IIIA with micro-metastasis size > $1 \mathrm{~mm}$, IIIB and IIIC) [18]. A study comparing the use of nivolumab in adjuvant therapy with a combination of nivolumab and ipilimumab is currently under way (CheckMate 915).

\section{Molecularly targeted treatment}

Adjuvant therapy using dabrafenib with trametinib in patients with high risk BRAF mutation level III showed improvement in RFS (HR 0.47), DMFS (HR 0.51; 91\% vs 70\% after 1 year, $77 \%$ vs $60 \%$ after 2 years and $71 \%$ vs $57 \%$ after 3 years) and OS (HR 0.57) compared to placebo. In this study (COMBI-AD) dabrafenib with tramethinib were used for 1 year compared to placebo (stage IIIA with a metastasis di meter $>1 \mathrm{~mm}$, IIIB/C) [19]. This study is the only one apart from the study with ipilimumab discussed above that has shown a significant improvement in overall survival rate. The safety profile of dabrafenib with tramethinib was consistent with that observed in the study, which included patients with melanoma at the IV stage of development. Dabrafenib with trametinib are currently approved for adjuvant therapy in the United States and the European Union. The formally 'positive' clinical trial BRIM-8 [20] also included the use of vemurafenib monotherapy in adjuvant therapy for one year as compared to placebo in patients with melanoma after stage IIC-III resection (the only study to date covering patients at stage II). The median disease-free survival (DFS) was 23.1 months in the vemurafenib-treated group compared to 15.4 months in the placebo group (HR 0.8; $p=0.026$ ), but this effect was limited only to the IIC-IIIA-IIIB subgroup, and was not visible to more advanced patients at stage IIIC. At the same time, it is known from the current practice at patients with metastatic melanoma that monotherapy with BRAF inhibitors is not optimal if compared to the combined treatment of patients with the presence of $B R A F$ mutations with BRAF and MEK inhibitors.

\section{Summary}

The results of systemic adjuvant therapy with immunotherapy after high-risk melanoma resection are summarised in Table II. Other immunotherapy methods (e.g. interleu- kin-2), vaccines or drugs with cytotoxic effects have no practical use in adjuvant postoperative therapy [1, 4, 5, 21].

In summary, adjuvant therapy with anti-PD-1 immunotherapy (ninolumab or pembrolizumab) or combined treatment with BRAF and MEK inhibitors (dabrafenib with tramethinib for populations with BRAF mutations) is becoming a new standard of care after melanoma resection with high risk of relapse (resection stages IIIA-IV) according to American and Polish recommendations $[2,4,22]$. This, in turn, means that the treatment of all patients with melanomas with stages from IIIA to IV should be discussed at multi-specialist team meetings in order to ensure optimal, modern and as effective a treatment as possible. In addition, it is important to ensure that high risk melanoma patients are included in prospective clinical trials of new adjuvant therapy where possible.

\section{Conflict of interest: none declared}

\section{Piotr Rutkowski, MD, PhD}

Maria Skłodowska-Curie Institute - Oncology Center Department of Soft Tissue/Bone Sarcoma and Melanoma ul. Roentgena 5, 02-781 Warszawa, Poland e-mail:piotr.rutkowski@coi.pl

Received: 23 Aug2018

Accepted: 28 Aug2018

\section{References}

1. Rutkowski P (ed.). Złośliwe nowotwory skóry. Via Medica: Gdańsk 2014.

2. Rutkowski P, Wysocki PJ (ed.). Cutaneous melanoma. Guidelines for diagnostic and therapeutic management. Oncology in Clinical Practice 2017; 13: 241-258.

3. Dummer $\mathrm{R}$, Hauschild A, Lindenblatt N et al. ESMO Guidelines Committee. Cutaneous melanoma: ESMO Clinical Practice Guidelines for diagnosis, treatment and follow-up. Ann Oncol 2015; 26 Suppl 5:v126-v132.

4. NCCN Guidelines. Melanoma, Version 3.2018.

5. Eggermont AMM, Dummer R. The 2017 complete overhaul of adjuvant therapies for high-risk melanoma and its consequences for staging and management of melanoma patients. Eur J Cancer 2017; 86: 101-105.

6. Ballo MT, Ang KK. Radiotherapy for cutaneous malignant melanoma: rationale and indications. Oncology 2004; 18: 99-107.

7. Burmeister $\mathrm{BH}$, Henderson MA, Ainslie J et al. Adjuvant radiotherapy versus observation alone for patients at risk of lymph-node field relapse after therapeutic lymphadenectomy for melanoma: a randomised trial. Lancet Oncol 2012; 13: 589-597.

8. Henderson MA, Burmeister BH, Ainslie J et al. Adjuvant lymph-node field radiotherapy versus observation only in patients with melanoma at high risk of further lymph-node field relapse after lymphadenectomy (ANZMTG 01.02/TROG 02.01): 6-year follow-up of a phase 3, randomised controlled trial. Lancet Oncol 2015; 16: 1049-1060.

9. Eggermont AM, Gore M. Randomized adjuvant therapy trials in melanoma: surgical and systemic. Semin Oncol 2007; 34: 509-515.

10. SondakVK, Gonzalez RJ, Kudchadkar R. Adjuvant therapy for melanoma: a surgical perspective. Surg Oncol Clin North Am 2011; 20: 105-114.

11. Eggermont AM, Suciu S, Testori $A$ et al. Ulceration and stage are predictive of interferon efficacy in melanoma: results of the phase III adjuvant trials EORTC 18952 and EORTC 18991. Eur J Cancer 2012; 48: 218-225.

12. Ives NJ, Suciu S, Eggermont AM et al.; International Melanoma Meta-Analysis Collaborative Group (IMMCG). Adjuvant interferon-a for the treatment of high-risk melanoma: An individual patient data meta-analysis. Eur J Cancer 2017; 82: 171-183.

13. Rutkowski P. Świtaj T. Immunoterapia czerniaków. In: Immunoonkologia. Wysocki P (ed.). Gdańsk: Via Medica, 2015.

14. Eggermont AM, Chiarion-Sileni V, Grob JJ et al. Adjuvant ipilimumab versus placebo after complete resection of high-risk stage III melanoma 
(EORTC 18071): a randomised, double-blind, phase 3 trial. Lancet Oncol 2015; 16: 522-530.

15. Eggermont AMM, Chiarion-Sileni V, Grob JJ et al. Prolonged survival in stage III melanoma with ipilimumab adjuvant therapy. NEng/ J Med 2016; 375: 1845-1855.

16. Weber J, Mandala M, Del Vecchio M et al.; CheckMate 238 Collaborators. Adjuvant nivolumab versus ipilimumab in resected stage III or IV melanoma. N Engl J Med 2017; 377: 1824-1835.

17. Weber JS, Mandalà $M$, Del Vecchio $M$ et al. Adjuvant therapy with nivolumab (NIVO) versus ipilimumab (IPI) after complete resection of stage III/IV melanoma: Updated results from a phase III trial (CheckMate 238). J Clin Oncol 2018; 36 (Suppl; abstr 9502). ASCO Annual Meeting.

18. Eggermont AMM, Blank CU, Mandala M et al. Adjuvant pembrolizumab versus placebo in resected stage III melanoma. NEng/ J Med 2018; 378: 1789-1801.
19. Long GV, Hauschild A, Santinami M et al. Adjuvant dabrafenib plus trametinib in stage III BRAF-mutated melanoma. N Engl J Med 2017; 377: 1813-1823.

20. Maio M, Lewis K, Demidov L et al.; BRIM8 Investigators. Adjuvant vemurafenib in resected, BRAFV600 mutation-positive melanoma (BRIM8): a randomised, double-blind, placebo-controlled, multicentre, phase 3 trial. Lancet Oncol 2018; 19: 510-520.

21. Dreno B, Thompson JF, Smithers BM et al. MAGE-A3 immunotherapeutic as adjuvant therapy for patients with resected, MAGE-A3-positive, stage III melanoma (DERMA): a double-blind, randomised, placebo-controlled, phase 3 trial. Lancet Oncol 2018; 19: 916-929.

22. Bello DM, Ariyan CE. Adjuvant therapy in the treatment of melanoma. Ann Surg Oncol 2018; 25: 1807-1813. 